J. Clin. Chem. Clin. Biochem.

Vol. 15, 1977, pp. 201-204

\title{
Eine Methode zur Bromidbestimmung im Harn mit Hilfe einer ionensensitiven Elektrode
}

\author{
Von J. Angerer \\ Aus dem Zentralinstitut für Arbeitsmedizin (Direktor: Prof. Dr. G. Lehnert, Ordinariat für Arbeitsmedizin der Uni- \\ versität Hamburg) der Gesundheitsbehörde der Freien und Hansestadt Hamburg
}

(Eingegangen am 27. September/5_November 1976)

Zusammenfassung: Es wird eine potentiometrische Methode zur Bestimmung der Bromidkonzentration im menschlichen Harn beschrieben. Wegen ihrer Spezifität und der erzielbaren Genauigkeit dieser Methode ist sie besonders zum Nachweis geringer Bromidkonzentrationen geeignet, wie sie als Folge beruflicher Bromalkanexpositionen zu erwarten sind. Die Wiederauffindungsraten betrugen 102,5-112,5\% mit einem VK zwischen 5,8 und 8,6\%. Die Nachweisgrenze betrug $0,013 \mathrm{mmol} / \mathrm{l}$. Bei einem Kollektiv von Normalpersonen $(n=20)$ wurden Bromidausscheidungen zwischen 0,059 und $0,162 \mathrm{mmol} / \mathrm{l}$ gefunden.

\section{$A$ method for the determination of bromide in urine with an ion-sensitive electrode}

Summary: A potentiometric method for determining the concentration of bromide in human urine is described. On account of its specificity and precision, this method is especially suited for the determination of slightly elevated bromide concentrations due to occupational exposure to alkyl bromides. The rate of recovery is 102.5 to $112.5 \%$, and the variability coefficients lie between 5.8 and $8.6 \%$. The detection limit is about $0.013 \mathrm{mmol} / \mathrm{l}$. The concentrations of bromide in the urine of a group of normal persons lie between 0.059 and $0.162 \mathrm{mmol} / 1$.

\section{Einleitung}

Bestimmungen dẹ Bromidausscheidung im Hạn sind nicht nur zum Nachweis einer mißbräuchlichen Einnahme bromureidhaltiger Schlafmittel, sondern auch zur Abklärung arbeitsmedizinischer Sachverhalte von Interesse, finden doch einige Bromderivate niedriger Alkane, insbesondere Methylbromid, z. B. als Fungizid, Kühlflüsssigkeit, etc. eine breite Anwendung (1). Eine zusammenfassende Darstellung der bei akuter und chronischer Einwirkung von Methylbromid auftretenden Gesund dheitsschäden hat kürzlich die Arbeitsstoffkommission der Deutschen Forschungṣgemeinschaft gegeben (2). Sie kommt abschließend zu der Ausssage, daß für den derzeitigen MAK $^{1}$ )-Wert von $78,92 \mathrm{mg} / \mathrm{m}^{3}$ eine Uberprüfung dringend erforderlich erscheint. Bei der toxikologischen Gesamtbewertung sollten entsprechend der Präàmbel zur MAK-Wertliste Erfahrungen am Menschen grundsätzlich Vorrang vor Tierversuchen haben. Eine Neubearbeitung der Problemàtik muß daher breit angelegte Felduntersuchungen am Arbeitsplatz implizieren. Ein einfaches, analytisch valides Verfahren zur Bestimmung geringer Konzentrationen

\footnotetext{
1) $\mathrm{MAK}=$ Maximale Arbeitsplatz-Konzentration.
}

von Bromid im biologischen Material ist hierzu Voraussetzung. Das hier beschriebene Verfahren vermag die apparativ aufwendigeren Methoden der Röntgenfluoreszenz- und der Neutronenaktivierungsanalyse in diesem Zusammenhang zu ersetzen.

\section{Methodik}

\section{Prinzip der Methode}

Die organische Matrix des Harns wird durch eine alkalische Veraschung nach Kisser (3) zerstört. Durch Umsetzung mit Kaliumpermanganat in schwach mineralsaurer Lösung wird das Bromid des Rückstandes selektiv zu Brom oxidiert. Letzteres wird in eine Vorlage übergetrieben und mit saurer Sulfitlösung wiederum zu Bromid reduziert. Nach dieser selektiven Abtrennung von anderen Harnbestandteilen läßt sich nun der Bromidgehalt mit Hilfe einer ionensensitiven Elektrode störungsfrei bestimmen.

Aufarbeitung des Harns und Abtrennung des Bromids in Form von Brom

$5 \mathrm{ml}$ Harn werden in einem Nickeltiegel mit $0,5 \mathrm{ml}$ wäßriger Natriumcarbonatlösung $(1 \mathrm{~mol} / \mathrm{l})$ versetzt und im Wärmeschrank bei $80^{\circ} \mathrm{C}$ zur Trockene eingedampft und dann etwa 4 Stunden bei $540^{\circ} \mathrm{C}$ verascht. Der weiße Rückstand wird anschließend in $5 \mathrm{ml}$ bidest. Wasser aufgenommen und unter 
Nachspülen mit rund $15 \mathrm{ml}$ bidest. Wasser in die Oxidationsapparatur (Abb. 1) übergefülurt. Nach Zugabe von $500 \mathrm{mg}$ Kaliumpermanganat verschließt man den Reaktionskolben und fügt aus dem Tropftrichter $3 \mathrm{ml}$ Schwefelsäure $(1 \mathrm{~mol} / \mathrm{l}) \mathrm{zu}$. Unter Erhitzen des Reaktionsgefäßes auf $80^{\circ} \mathrm{C}$ leitet man einen Stickstoffstrom von 0,31/min durch das Reaktionsgemisch. Die entstehenden bromhaltigen Dämpfe werden in eine Vorlage geleitet, die $15 \mathrm{ml}$ wäßrige Natriumsulfitlösung $(0,05 \mathrm{~mol} / 1)$ und $0,5 \mathrm{ml}$ Schwefelsäure $(1 \mathrm{~mol} / \mathrm{l})$ enthält (pH 2,0). Nach 15 Minuten ist die Reaktion beendet, und man versetzt die Vorlage mit $2 \mathrm{ml}$ eines Ionenstärkeausgleich-puffers, der $125 \mathrm{~g} \mathrm{KNO}_{3} / 1$ enthält. Dann füllt man die Lösung auf $25 \mathrm{ml}$ auf und stellt sie nach dem Überführen in ein 50-ml-Becherglas am pH-Meter mit einigen Tropfen Natronlauge $(1 \mathrm{~mol} / \mathrm{l})$ auf $\mathrm{pH} 2,5$ ein.

\section{Potentiometrische Endpunktbestimmung}

In die mittels eines Magnetrührers gemischte Bromidlösung taucht man die bromidspezifische $\mathrm{AgBr} / \mathrm{Ag}_{2} \mathrm{~S}$-Festkörperelektrode (ORION 94-35), sowie die mit Kaliumchloridlösung (ORION 90-00-01) gefüllte Ag/AgCl-Elektrode (Orion 90-01) ein, die als Bezugselektrode dient. An einem mV-Meter mit gespreizter Skala (ORION; Typ 701) wird die Potentialdifferenz nach 2 Minuten abgelesen. Wegen der Temperaturabhängigkeit der Elektrodenpotentiale sollten die Untersuchungs- bzw. Standardlösungen auf gleiche, konstante Temperatur, z. B. $25^{\circ} \mathrm{C}$ gebracht werden.

\section{Kalibrierung der Meßanordnung}

Vor jeder Analysenserie wird bei gleicher Meßanordnung die Anzeige des $\mathrm{mV}$-Meters mit Hilfe einer frisch hergestellten Natriumbromidstandardlösung auf einen konstanten Wert (z. B. $150 \mathrm{mV}$ ) eingestellt. Zur Herstellung dieser Lösung werden $5 \mathrm{ml}$ einer Natriumbromidlösung $(0,125 \mathrm{mmol} / \mathrm{l})$ mit $2 \mathrm{ml}$ Kaliumnitratlösung versetzt, die $1,25 \mathrm{~mol} / \mathrm{l}$ enthält, und mit bidest. Wasser auf $25 \mathrm{ml}$ aufgefüllt. Am pH-Meter wird diese Lösung mit einigen Tropfen verdünnter Schwefelsäure auf $\mathrm{pH} 2,5$ eingestellt.

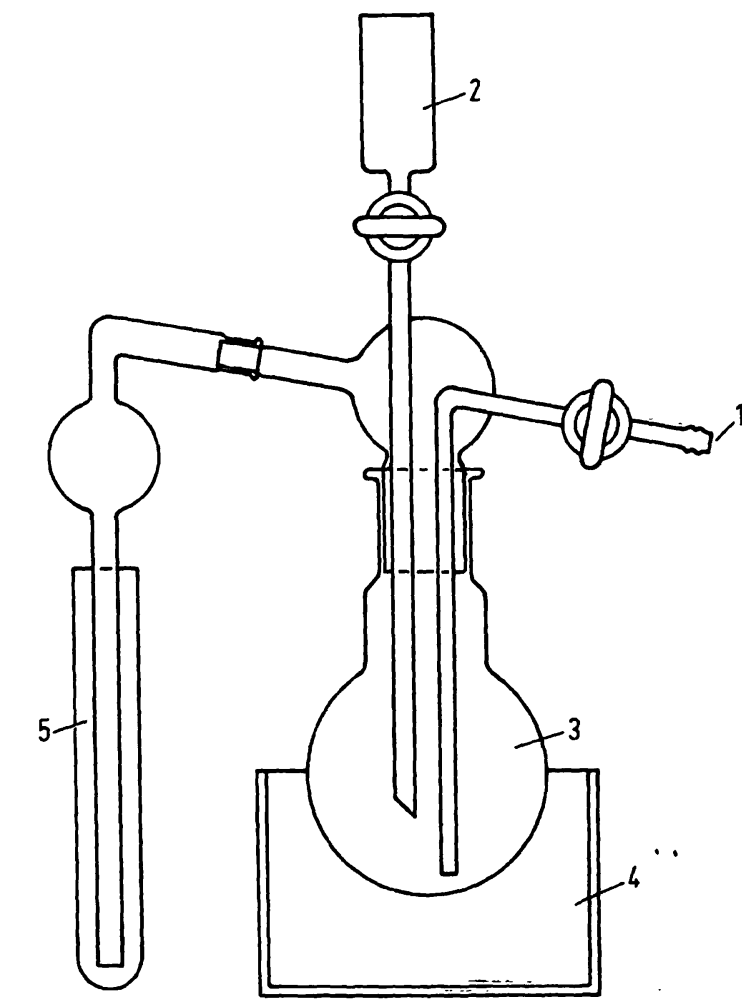

Abb. 1. Schema der Oxidationsapparatur.

1 Stickstoffeinleitungsrohr; 2 Tropf trichter für Schwefelsäure; 3 Reaktionskolben; 4 Ölbad; 5 Vorlage mit saurer Sulfitlösung.

\section{Kalibrierung - Berechnung der Analysenergebnisse}

Zur Erstellung von Standardkurven werden wäßrige Bromidlösungen hergestellt, die zwischen 0,06 und $12,5 \mathrm{mmol} \mathrm{NaBr} / 1$ Wasser enthalten. Diese Lösungen werden wie Harnproben aufgearbeitet und vermessen. Auf halblogarithmischem Papier trägt man die gemessenen Elektrodenpotentiale auf der linearen Achse, die eingesetzten Bromidkonzentrationen auf der logarithmischen Achse auf. Mit den für die Harnproben gemessenen Potentialen geht man in diese Standardkurve ein und liest die zugehörigen Konzentrationswertè für Bromid ab.

\section{Ergëbnisse}

Die Standardkurve erweist sich im Bereich zwischen 0,06 und $12,5 \mathrm{mmol}$ Bromid/l als linear. In Übereinstimmung mit der Literatur ändert sich das Elektrodenpotential um $57,5 \mathrm{mV}$ bei einer Änderung der Bromidkonzentration um das lofache (4).

Weil die bromidsensitive Elektrode Querempfindlichkeiten gegen stark reduzierende Substanzen, sowie vor allen gegenüber Anionen aufweist, die mit Silber schwerlösliche Salze bilden, wurde die Spezifität der Methode in Vorversuchen überprüft. Naturgemäß kam dabei den physiologischerweise im Harn vorkommenden Chlorid-, Jodidund Cyanidionnen die größte Bedeutung zu. Wäßrige Lösungen dieser Störsubstanzen wurden deshalb wie beschrieben aufgearbeitet und vermessen. Es zeigte sich, daß selbst Konzentrationen, die das 10 fache der normalerweise im Harn vorkommenden betragen (5), das Ergebnis der Bromidbestimmung nicht beeinträchtigen.

Die Genauigkeit und Richtigkeit der Methode wurde durch mehrmalige Analyse von Harnproben getestet, denen unterschiedliche Bromidmengen zugesetzt worden waren. Die Mittelwerte $(\overline{\mathrm{x}})$, Standardabweichungen (s) und Variationskoeffizienten (VK) dieser Bestimmungen sowie die unter Berücksichtigung des Harnleerwertes errechneten Wiederfindungsraten sind in Tabelle 1 zusammengefaßt.

Die Nachweisgrenze der beschriebenen Bromidbestimmungsmethode beträgt unter den angegebenen Analysenbedingungen rund $0,013 \mathrm{mmol} / 1 \mathrm{Harn}$.

Die Anwendbarkeit dièser Methode zur Bestimmung physiologischer Bromidkonzentrationen haben wir an einem kleinen Kollektiv $(n=20)$ von unbèlasteten Personen überprüft.

Tab. 1. Genauigkeit und Richtigkeit von Bromidbestimmungen im Harn.

\begin{tabular}{llllll}
\hline $\mathbf{n}$ & $\begin{array}{l}\text { Sollwert } \\
\text { (Leerwert }+\mathrm{Zu}- \\
\text { gabe) } \\
{[\mathrm{mmol} / \mathrm{l}]}\end{array}$ & $\begin{array}{l}\text { Istwert } \\
\text { [mmol/l] }\end{array}$ & $\begin{array}{l}\text { s } \\
{[\mathrm{mmol} / \mathrm{l}]}\end{array}$ & $\begin{array}{c}\text { VK } \\
{[\%]}\end{array}$ & $\begin{array}{l}\text { Wieder- } \\
\text { findung }\end{array}$ \\
\hline 8 & $0,095+0$ & 0,095 & 0,006 & 7,5 & - \\
8 & $0,095+0,125$ & 0,215 & 0,014 & 5,8 & 112,5 \\
8 & $0,095+0,250$ & 0,375 & 0,024 & 6,9 & 102,5 \\
8 & $0,095+1,250$ & 1,471 & 0,127 & 8,6 & 109,1 \\
\hline
\end{tabular}


Tab. 2. Gemessene Bromidkonzentrationen in Harnproben eines Normalkollektivs $(n=20)$.

\begin{tabular}{|c|c|c|c|}
\hline $\begin{array}{l}\text { Proband } \\
\text { Alter } \\
\text { Geschlecht }\end{array}$ & $\begin{array}{l}\text { Bromid- } \\
\text { konzentration } \\
\text { [mmol/l] }\end{array}$ & $\begin{array}{l}\text { Proband } \\
\text { Alter } \\
\text { Geschlecht }\end{array}$ & $\begin{array}{l}\text { Bromid- } \\
\text { konzentration } \\
\text { [mmol/I] }\end{array}$ \\
\hline $\begin{array}{l}25 \% \\
33 \% \\
50 \% \\
26 \% \\
50 \% \\
22 \% \\
43 \% \\
33 \% \\
48 \% \\
19 \%\end{array}$ & $\begin{array}{l}0,130 \\
0,102 \\
0,108 \\
0,083 \\
0,100 \\
0,089 \\
0,102 \\
0,094 \\
0,087 \\
0,139\end{array}$ & $\begin{array}{l}518 \\
428 \\
528 \\
548 \\
348 \\
37 \% \\
378 \\
358 \\
618 \\
150\end{array}$ & $\begin{array}{l}0,121 \\
0,145 \\
0,077 \\
0,074 \\
0,162 \\
0,138 \\
0,118 \\
0,074 \\
0,099 \\
0,059\end{array}$ \\
\hline
\end{tabular}

Die männlichen und weiblichen Personen (15-61 Jahre) gaben an, während der letzten beiden der Untersuchung vorausgehenden Wochen keine bromidhaltigen Medikamente, insbesondere keine Schlafmittel zu sich genommen zu haben. Es wurden Bromidausscheidungen zwischen 0,059 und $0,162 \mathrm{mmol} / \mathrm{l}$ gefunden. Die mittlere Ausscheidung betrug $0,105 \pm 0,027 \mathrm{mmol} / 1$. Die gemessenen individuellen Bromidausscheidungen im Harn sind in Tabelle 2 aufgeführt.

\section{Diskussion}

Strenge arbeitssicherheitstechnische Bestimmungen für den Umgang mit niedrigen Bromalkanen lassen auch bei beruflicher Exposition Bromidausscheidungen erwarten, die nur wenig über den physiologischen Konzentrationen liegen. Die für den Bereich der arbeitsmedizinisch-toxikologischen Analy tik notwendigen Verfahren zur Bromidbestimmung im biologischen Material müssen deshalb spezifisch, empfindlich und genau sein. Stärker streuende Analysenergebnisse würden nämlich die Objektivierung der zu erwartenden geringfügigen Änderungen der Bromidausscheidung verhindern.

Zwar ist in der Literatur bereits eine Reihe von Bromidbestimmungsme thoden beschrieben, doch werden die wenigsten den eingangs erhobenen Forderungen gerecht.

Die photometrischen Methoden, die auf der Bildung von gefärbten Goldbromiden $(6,7)$ bzw. auf der Umsetzung von Brom mit Pararosanilin (8) bzw. Fluoresceîn (9) beruhen, sind für den Nachweis physiologischer bzw. arbeitsmedizinisch-relevanter Bromidkonzentrationen zu unempfindlich und ungenau ( 3 , 10). Dies gilt auch für die in der forensischen Toxikologie weit verbreitete Phenolrotmethode nach Goldman (11) und Kisser (3), die sich gut zum Nachweis erhöhter Bromidausscheidungen eignet. Auch das auf der von Street (12) beschriebenen Methode aufbauende gaschromatographische Verfähren von Archer (13) eignet sich nicht zum Nachweis kleiner Bromidkonzentrationen. Eine kritische Betrachtung der älteren Bromidbestimmungsmethoden findet sich u. a bei Hinsberg (14) und Beyer (15). Grundsätzlich ermöglicht die Anwendung der modernen physikalischen Verfahren der Röntgenfluoreszenzanalyse $(16,17$,
$18,19,20,21,22,23)$ und der Neutronenaktivierungsanalyse $(24,17)$ eine analytisch valide Bromidbestimmung im biologischen Material. Besonders die Neutronenaktivierungsanalyse wird wegen ihrer erwiesenen Vorteile gerne als Referenzmethode eingesetzt (17). Um allerdings im Bereich physiologischer Bromidkonzentrationen genaue und richtige Ergebnisse zu erhalten, erfordern aucli diese Methoden sehr aufwendige Probenaufarbeitungen. Bei ihnen lassen sich - wie bei den meisten der angeführten Verfahren - Matrixeffekte, die die Messung kleiner Bromidkonzentrationen empfindlich zu stören vermögen, am wirkungsvollsten durch eine Veraschung des Probenmaterials eliminieren $(18,19,21)$. Es wurde auch versucht, den Störuntergrund durch die Erstellung von Standardkurven in der jeweiligen biologischen Matrix $(17,21)$ bzw. durch die Verwendung eines inneren Standards (18) zu berücksichtigen. Ohne Einbuße an analy tischer Validität sind diese Vorkehrungen sicherlich bei der Messung erhöhter, nicht jedoch physiologischer Bromidkonzentrationen möglich.

Trotz verschiedener Versuche $(25,26)$ konnten sich ionensensitive Elektroden wegen ihrer Querempfindlichkeiten noch nicht zur Bestimmung von Bromid im biologischen Material durchsetzen. Hauptsächlich der im Vergleich zu Bromid 1000 fache Chloridüberschuß in den Körperflüssigkeiten aber auch Jodid, Cyanid und Cystein (26) verhinderten bisher die Erstellung einer praktikablen und analytisch validen Bromidbestimmungsmethode mit Hilfe ionensensitiver Elektroden.

Die Schwierigkeiten bei der Bestimmung kleiner Bromidkonzentrationen im biologischen Material, die zumeist auf eine mangelnde Spezifität des Bestimmungsverfahrens und auf Matrixeffekte zurückgehen, konnten bei der hier beschriebenen Methode beseitigt werden. Durch eine Oxidation des Veraschungsrückstandes mit Kaliumpermanganat in schwach mineralsaurer Lösung wird das Bromid als flüchtiges Brom selektiv von der störenden biologischen Matrix abgetrennt. In Kombinationen mit der anschließenden Endpunktsbestimmung mittels einer bromidsensitiven Elektrode bewirkt dieser Abtrennungsschritt die Spezifität der Methode. Darüber hinaus entfällt der analytische Störuntergrund, und es wird sowohl die Erfassung wie die hinreichend genaue Bestimmung physiologischer Bromidkonzentrationen im Harn ermöglicht. Selbstverständlich lassen sich auch erhöhte Bromidkonzentrationen zuverlässig erfassen (Tab. 1).

Die unter den angegebenen methodischen Bedingungen erzielte Nachweisgrenze von rund $0,013 \mathrm{mmol} / \mathrm{l} \mathrm{Harn}$ ermöglicht sowohl die Bestimmung physiologischer wie arbeitsmedizinisch zu erwartender Bromidkonzentrationen. Da die Nachweisgrenze praktisch nur vom Nachweisvermögen der bromidsensitiven Elektrode begrenzt wird, könnte sie bei Bedarf durch eine Variation der Probenaufarbeitung weiter gesenkt werden.

Natürlich ist die Praktikabilität der Methode durch die zeitintensive Trockenveraschung begrenzt. Gleichwohl kann auf sie nach unseren Erfahrungen nicht verzichtet werden, wenn physiologische Bromidmengen nachzuweisen sind. Da in diesem Fall jedoch auch die apparativen ungleich aufwendigeren Methoden der Röntgenfluoreszenz- und der Neutronenaktivierungsanalyse nicht auf eine Veraschung verzichten können, stellt unser Verfahren sicherlich einen methodischen Fortschritt dar. Auch im Hinblick auf zum Teil apparativ 
aufwendigere Bromidbestimmungsverfahren erscheint die analytische Zuverlässigkeit der Methode durchaus zufriedenstellend, ohne wie im Falle der Röntgenfluoreszenz-oder der Neutronenaktivierungsanalyse teure Gerätekapazität in Anspruch zu nehmen.

Ein Vergleich der hier für das unbelastete Kollektiv ermittelten Bromidkonzentrationen mit entsprechenden veröffentlichten Daten ist aus methodischen Gründen (Altersstruktur, Ausscheidungsraten statt Konzentrationsangaben etc.) nur bedingt möglich. Immerhin zeigen diese Ergebnisse eine gute Übereinstimmung mit den im Vergleich zur Vielzahl der veröffentlichten Bromidbestimmungsmethoden spärlichen Angaben in der Literatur (5, $22,24)$. Die hier gewonnenen Werte sollen nur die grund- sätzliche Brauchbarkeit der Methode zeigen, nicht jedoch verbindliche Normwerte festlegen. Hierzu bedarf es der Untersuchung einer größeren Stichprobe unter definierten Bedingungen.

\section{Danksagung}

Mein besonderer Dank gilt Frau $I$. Wendländer für Ihre äußerst engagierte Mitarbeit bei der Erarbeitung der Methode. Frau I. Groth-Tittmann danke ich für Ihrue Unterstützung bei der Literaturarbeit.

Diese Arbeit wurde mit Mitteln der Deutschen Forschungsgemeinschaft unterstützt.

\section{Literatur}

1. Encyclopaedia of Occupational Health and Safety. (1971), I, 213-214, International Labour Office, Genf.

2. Henschler, D. (1973), Gesundheitsschädliche Arbeitsstoffe. Hrsg. Deutsche Forschungsgemeinschaft, Verlag Chemie $\mathrm{GmbH}$, Weinheim.

3. Kisser, W. (1967), Arch. Toxikol. 22, 404-409.

4. Camman, K. (1973), Das Arbeiten mit ionenselektiven Elektroden, Springer Verlag, Berlin-Heidelberg-New York.

5. Geigy, (1968), Wissenschaftliche Tabellen, 7. Aufl., S. 658 bis 661 .

6. Barbour, R. F., Pilkington, F. \& Sargent, W. (1936), Brit. Med. J. 957-962.

7. Underwood, P. J. (1967), Aust. J. Exp. Biol. Med. Sci. $45,577-580$.

8. Hunter, G. \& Goldspink, A. A. (1955), Biochem. J. 60 , 261-264.

9. Sommerville, W. C. \& Campbell, A. D. (1963), Mikrochim. Acta 991-992.

10. Kamm, G. \& Schrinner, G. (1969), Beitr. Gerichtl. Med. 25, 293-299.

11. Goldman, E. \& Byles, D. (1959), J. Amer. Water Works Ass. $51,1051-1056$.

12. Street, H. V. (1960), Clin. Chim. Acta 5, 938-943.
13. Archer, A. W. (1972), Analyst 97, 428-432.

14. Hinsberg, K. \& Lang, K. (1957), Medizinische Chemie für den klinischen und theoretischen Gebrauch. Urban und Schwarženbach. München-Ber̂lin-Wien.

15. Beyer, K.-H. (1971), Pharm. Ztg. 47, 1803-1806.

16. Mathies, J. C. \& Lund, P. K. (1960), Norelco Reporter 7, 134-139.

17. Beyermann, K. (1961), Z. Anal. Chem. 183, 199-203.

18. Herrmann, M. (1961), Z. Anal. Chem. 181, 122-125.

19. Natelson, S., Sheid, B. \& Leighton, D. R. (1962), Clin. Chem. 8, 630-639.

20. Cretius, K. \& Beyermann, K. (1962), Klin. Wochenschr. 40, 89-97.

21. Hauck, G. (1968), Z. Anal. Chem. 243, 98-103.

22. Hauck, G. (1968), Arch. Toxikol. 23, 273-282.

23. Purdham, J. T. \& Strausz, O. P. (1975), Anal. Chem. 47, 2030-2032.

24. Heurtebise, M. \& Ross, W. J. (1971), J. Radioanal. Chem. $8,5-12$.

25. Degenhardt, H. J., Abeln, G., Bevaart, B. \& Baks, J (1972), Clin. Chim. Acta 38, 217-220.

26. Poser, S., Poser, W. \& Müller-Oerlinghausen, B. (1974), diese Z. 12, 350-351.
Dr. J. Angerer

Zentralinstitut für Arbeitsmedizin Adolph-Schönfelder-Straße 5 D-2000 Hamburg 76 